\title{
Ordem do Nascimento e Abertura à Experiência: Comunicação Breve
}

\author{
Silvio José L. Vasconcellos * \\ Universidade Federal de Santa Maria, Santa Maria, Brasil \\ Felipe Valentini \\ Universidade de Brasília, Brasília, Brasil \\ Nelson Hauck Filho \\ Cláudio S. Hutz \\ Universidade Federal do Rio Grande do Sul, Porto Alegre, Brasil
}

\begin{abstract}
RESUMO
Ordem do nascimento é definida como a posição ocupada por uma pessoa conforme a sua idade entre seus irmãos e irmãs. Abertura à experiência é uma das cinco dimensões básicas da personalidade. $\mathrm{O}$ objetivo desta pesquisa foi investigar a relação entre ordem do nascimento e abertura à experiência em uma amostra de 472 estudantes universitários (média de idades $=24,6$ anos; $D P=8,8$ ). Os resultados indicaram efeitos distintos para homens e mulheres quanto à influência da ordem do nascimento. Os resultados são discutidos em termos de possíveis particularidades culturais e do delineamento metodológico empregado para a avaliação da personalidade.
\end{abstract}

Palavras-chave: personalidade; abertura à experiência; ordem do nascimento.

\begin{abstract}
Birth Order and Openness to Experience: Brief communication

Birth order is defined as a person's rank by age among his or her siblings. Openness to experience is one of five major domains of personality. The purpose of this research was to investigate the relationship between birth order and openness to experience in a sample of 472 university students (mean age $=24.6$ years; $S D=8.8$ ). Results showed distinct effects for men and women regarding the influence of birth order. Authors discuss the results in light of possible cultural features and of the personality assessment method employed.
\end{abstract}

Keywords: personality; openness to experience; birth order.

A expressão "ordem do nascimento" refere-se à posição ocupada pelos diferentes irmãos em uma mesma família conforme a idade de cada um. A influência da ordem do nascimento no desenvolvimento da personalidade revela-se uma das questões mais controvertidas da Psicologia moderna, considerando a não convergência dos resultados até então alcançados em pesquisas voltadas para o assunto. O psiquiatra austríaco Alfred Adler foi um dos primeiros teóricos a presumir que posições ocupadas nessa ordem acabam exercendo influências sobre a personalidade do indivíduo, ainda que não tenha realizado pesquisas controladas para avaliar tais influências. Para o autor, o filho primogênito tenderia a ser "destronado" pelos próprios irmãos que nascem depois dele (Adler, 1964). Posteriormente, diversos autores buscaram abordar o tema empiricamente, sendo que Beer e Horn (2000) encontraram 1729 obras publicadas ou citadas no Journal of Individual Psychology apenas entre os anos de 1963-1993. Problemas metodológicos relacionados

* Endereço para correspondência: Silvio José L. Vasconcellos - silviojlvasco@hotmail.com 
à interação de outras variáveis, a exemplo do tipo de relação estabelecida com os pais e idade dos mesmos também têm sido destacados na literatura sobre o tema (Dunkel, Harbke \& Papini, 2009). No Brasil, apesar de existirem trabalhos sobre o tema contemplando a avaliação de adolescentes (Sampaio \& Vieira, 2009), estudos comparativos com gêmeos (Tavares, 2004), a investigação da relação entre estilos interpessoais e ordem do nascimento (Magalhães, 2009), bem como a relação dessa variável com práticas educativas parentais (Sampaio, 2007), ainda há uma escassez de estudos abordando características específicas da personalidade e ordem do nascimento.

No que se refere aos estudos voltados para a exploração de diferenças entre filhos primogênitos e não primogênitos, destaca-se um significativo emprego do modelo dos Cinco Grandes Fatores (CGF) da personalidade na investigação dessas diferenças (Sulloway, 1999). O referido modelo tem como base uma série de estudos transculturais que, por intermédio da análise fatorial, apontam uma solução de cinco fatores para descrever e compreender a personalidade. Conforme descreveram Hutz et al. (1998), esses fatores denominam-se: Extroversão (i.e., o quanto uma pessoa é voltada para obter estimulação externa e procura estar em evidência diante dos demais indivíduos), Socialização (i.e., o quanto uma pessoa é altruísta e amigável), Realização (i.e., o quanto uma pessoa é responsável e dedicada), Neuroticismo (i.e., o quanto uma pessoa é aborrecida e ansiosa) e Abertura à Experiência (i.e., o quanto uma pessoa é criativa, imaginativa e investigativa). Esse último fator pode estar relacionado ainda a posições políticas, adesão a movimentos de vanguarda artística e maior receptividade a inovações em diferentes áreas.

Em um estudo de meta-análise abarcando 196 trabalhos prévios sobre a relação entre a ordem de nascimento e os CGF (Sulloway, 1995), verificou-se que filhos primogênitos tendem a ser mais extrovertidos, menos sociáveis, mais realizadores, mais neuróticos e menos abertos a novas experiências quando comparados a seus irmãos mais novos. Uma possível interpretação para essas diferenças é que elas representam o resultado de uma competição entre irmãos na tentativa de obter um maior grau de investimento paterno (Sulloway, 1999). Desse modo, os filhos primogênitos podem mostrar-se mais identificados com os pais e preocupados em satisfazer às expectativas dos mesmos, do que decorre serem mais neuróticos e conservadores (Sulloway, 1999). Em virtude disso, a personalidade pode estar relacionada ao modo como o indivíduo orienta-se e estabelece relações em seu nicho familiar.

Em contrapartida, autores como Harris (2000, 2007) indicam que a plasticidade neural, que caracteriza a espécie humana e permite respostas adaptativas orientadas pelas experiências individuais, somada à própria carga genética do indivíduo, faz com que a ordem do nascimento tenha uma influência muito restrita na formação da personalidade. De acordo com essa outra perspectiva evolucionista, aquilo que é aprendido a partir das relações que se estabelecem no contexto familiar, não é necessariamente transferido para outros contextos (Harris, 2000).

Segundo Harris (2000), a criança está apta a aprender outras formas de interação social, aplicáveis, por sua vez, ao ambiente que está para além do seu próprio nicho familiar. Por exemplo, um estudo evidenciou que algumas estratégias de dominação empregadas por filhos primogênitos diante dos seus irmãos menores não se manifestavam nas interações que se realizavam em outros contextos (Abramovitch, Corter, Pepler \& Stanhope, 1986). Além disso, alguns estudos também mostraram escassas evidências de diferenças entre primogênitos e não primogênitos quanto aos CGF (e.g., Jefferson, Herbst \& McCrae, 1998; Skinner, 2003).

No intuito de aprofundar essa discussão, o presente estudo procurou investigar as diferenças entre primogênitos e não primogênitos no que se refere à dimensão abertura à experiência dos CGF em uma amostra de estudantes universitários brasileiros. O estudo buscou trazer uma contribuição à área da investigação sobre os fatores que influenciam na expressão dos traços de personalidade sendo esse o principal objetivo do trabalho proposto. Tendo em vista o fato de que a influência do ambiente compartilhado na personalidade pode ser moderada por aspectos da cultura dos indivíduos (Bouchard \& Loehling, 2001), o presente estudo buscou apresentar uma investigação no contexto brasileiro no que se refere ao fator abertura à experiência. Optou-se por uma avaliação enfocando exclusivamente o citado fator, considerando ser essa uma dimensão capaz de explicar também a adesão ou rejei- 
ção a reformas políticas e culturais em diferentes contextos (Sulloway 1995, 1999). Além disso, uma comparação entre os gêneros, tal como a que está perfazendo o presente trabalho, permite que outros dados sejam agregados a análises já desenvolvidas em nosso país sobre esse tema (Samapio \& Vieira, 2010).

\section{MÉTODO}

\section{Participantes}

Participaram deste estudo 472 alunos de graduação de diferentes cursos da Universidade Federal do Rio Grande do Sul (UFRGS) e da Universidade Regional Integrada (URI), sendo 50,42\% do sexo masculino. Compuseram a amostra estudantes de Psicologia, Administração, Direito, Engenharia Civil e Engenharia Elétrica. A idade variou de 18 a 58 anos $(M=24,6$ e $D P=8,8)$. Os dados fazem parte de um estudo maior relacionado à tese de doutorado do primeiro autor, sendo que o $n$ amostral foi obtido selecionando-se apenas indivíduos que informaram ter pelo menos um irmão ou irmã.

\section{Instrumentos}

Utilizou-se, no presente estudo, a Escala Fatorial de Abertura à Experiência, elaborada e validada no Brasil por Vasconcellos e Hutz (2008). Esse instrumento psicométrico de autorrelato é constituído por 42 itens agrupados em três fatores (facetas de personalidade) distintos, denominados: Hábitos e Valores (17 itens; e.g., "Não gosto de situações que me causem surpresa"), Fantasia (nove itens; e.g., "Costumo pensar em soluções pouco convencionais para os problemas com os quais me deparo") e Atitudes (16 itens; e.g., "Gosto de fazer coisas que nunca fiz antes"). O instrumento foi avaliado em escala Likert de sete pontos $(1=$ Discordo totalmente, $7=$ Concordo totalmente). Os alphas de Cronbach se mostraram adequados para as escalas, Hábitos e Valores $=0,79$, Fantasia $=$ 0,73 e Atitudes $=0,81$. Também foi utilizado um questionário construído pelos pesquisadores, com o objetivo de avaliar aspectos sociodemográficos dos participantes, como o sexo, a idade, o número total de irmãos ou irmãs mais velhas (caso não fosse filho ou filha única), e se eram, ou não, primogênitos ou primogênitas.

\section{Procedimentos}

As coletas de dados foram realizadas nas cidades de Porto Alegre e Erechim, nas universidades anteriormente mencionadas. Todas as coletas ocorreram de forma padronizada, coletiva e em sala de aula, após explicações sobre os objetivos da pesquisa e assinaturas de termos de consentimento. O projeto de pesquisa foi previamente aprovado pelo Comitê de Ética em Pesquisa da Universidade Federal do Rio Grande do Sul.

\section{Análise dos dados}

Foram utilizadas estatísticas descritivas, testes $t \mathrm{e}$ análises de variância univariadas (ANOVA) e multivariadas (MANOVA). O software utilizado foi o SPSS 16.0.

\section{RESULTADOS}

Primeiramente, foram calculadas médias para os participantes nas facetas de personalidade da Escala Fatorial de Abertura à Experiência e no total da escala (média dos escores nos três fatores). Na Tabela 1, são apresentadas essas médias considerando o sexo dos participantes e a separação entre indivíduos primogênitos e não primogênitos. É possível ver que as estimativas pontuais de tendência central, bem como os desvios-padrão obtidos foram bastante semelhantes para cada variável entre os grupos. A seguir, uma Análise Multivariada da Variância (MANOVA) foi utilizada para investigar os efeitos do sexo e da ordem do nascimento em uma combinação linear das facetas de personalidade da Escala Fatorial de Abertura à Experiência. Assumiu-se a homogeneidade das variâncias, Box's $M(18,354080)=19,21, p=0,40$. Nenhum dos casos foi considerado influente (Distâncias de Cook e Leverage < 1), respaldando a utilização da MANOVA. Não foram encontradas diferenças entre os participantes primogênitos e não primogênitos no que se refere às médias da combinação linear das facetas de personalidade, Wilk's $\Lambda=99, F(3,409)=$ $1,04, p=0,37, \eta^{2}=0,01$. O efeito de interação entre o sexo e a ordem do nascimento também não foi significativo, Wilk's $\Lambda=0,99, F(3,409)=1,57, p=0,20, \eta^{2}$ $=0,01$. 
Tabela 1

Aspectos Descritivos dos Escores da EFA para Primogênitos e Não Primogênitos

\begin{tabular}{lcccc}
\hline \multirow{2}{*}{ Fatores da EFA } & \multicolumn{2}{c}{ Homens [Média $(D P)]$} & \multicolumn{2}{c}{ Mulheres [Média $(D P)]$} \\
\cline { 2 - 5 } & $\mathrm{NP}(n=152)$ & $\mathrm{P}(n=82)$ & $\mathrm{NP}(n=152)$ & $\mathrm{P}(n=82)$ \\
\hline Fantasia & $3,76(1,09)$ & $3,66(1,03)$ & $3,29(1,08)$ & $3,34(1,11)$ \\
Hábitos e Valores & $4,40(0,76)$ & $4,40(0,82)$ & $4,10(1,13)$ & $4,37(0,90)$ \\
Atitudes & $5,50(0,70)$ & $5,34(0,82)$ & $5,32(0,89)$ & $5,34(0,80)$ \\
Escore total & $4,71(0,48)$ & $4,58(0,53)$ & $4,40(0,57)$ & $4,48(0,52)$ \\
\hline Nota. NP = Não primogênitos; $P$ = Primogênitos; Os valores de ' $n$ ' sofreram pequenas alterações entre os fatores da EFA em decorrência dos casos ausentes (missing).
\end{tabular}

Subsequentemente, análises univariadas de variância (ANOVA) foram realizadas para avaliar os efeitos principais do sexo e da ordem do nascimento em cada faceta de personalidade do instrumento. Não foram encontrados efeitos principais significativos $(p<0,05)$, embora tenha sido possível verificar um efeito de interação entre o sexo e a ordem do nascimento para o escore total na Escala Fatorial de Abertura à Experiência, $F(3,409)=4,08, p=0,04, \eta^{2}=0,01$. Na sequência, foram realizados contrastes planejados (testes $t$ ) para as médias de primogênitos e não primogênitos nas facetas da abertura à experiência para cada sexo. Os resultados mostraram diferenças significativas entre homens primogênitos e não primogênitos para a escala de Atitudes, $t(470)=2,28, d=0,21, p=0,023$, e para o escore total no instrumento, $t(470)=2,79, d=$ $0,26, p=0,005$. Especificamente, homens não primogênitos apresentaram escores mais altos do que os primogênitos em ambas as variáveis. Além disso, mulheres primogênitas apresentaram escores mais altos na escala de Hábitos e Valores do que mulheres não primogênitas, $t(470)=2,87, d=0,27, p=0,004$.

\section{DISCUSSÃO}

Os dados obtidos não mostraram diferenças significativas entre primogênitos e não primogênitos para o total da amostra universitária utilizada. Todavia, um efeito de interação entre a ordem de nascimento e o sexo dos participantes chamou a atenção para a possibilidade de pequenas diferenças de tendência considerando a variável sexo. Contrastes planejados para cada sexo revelaram pequenos tamanhos de efeito, embora significativos, para a influência da ordem do nascimento na expressão do traço de personalidade abertura à experiência. Enquanto a tendência para os indivíduos do sexo masculino foi de primogênitos com es- cores mais baixos na faceta Atitudes e no escore total no instrumento - acompanhando os resultados da meta-análise de Sulloway (1995) -, o mesmo não ocorreu para os indivíduos do sexo feminino. A saber, mulheres primogênitas apresentaram escores mais altos para Hábitos e Valores do que aquelas não primogênitas. Ressalta-se, entretanto, um tamanho de efeito pequeno diante do qual os dados necessitam ser interpretados de forma ponderada.

Esse efeito moderador pode ser sugestivo de diferenças culturais específicas do contexto brasileiro. É possível, dessa forma, que os tipos de interação estabelecidos entre primogênitos, não primogênitos e seus pais sejam minimamente distintos para homens e mulheres. Resultados esses que sugerem uma concordância parcial com o trabalho de Sampaio e Vieira (2009), embora o presente estudo esteja restrito à avaliação do fator abertura à experiência. Desse modo, ainda que os estudos mostrem que o ambiente compartilhado explica muito pouco da variabilidade da personalidade (cf. Bouchard \& Loehling, 2001), fenômenos epigenéticos mediados pelo contexto cultural podem ser parcialmente responsáveis por modificar a expressão da abertura à experiência de forma distinta em homens e mulheres brasileiros. Além disso, infere-se que alguns desses fenômenos podem estar parcialmente relacionados à ordem do nascimento, embora os dados anteriormente destacados sejam insuficientes para afirmações mais peremptórias nesse sentido.

Outro aspecto a ser futuramente avaliado é uma possível influência moderadora do método de coleta dos dados (fonte de informação, se autorrelato ou informação de outras pessoas) na relação entre a ordem do nascimento e a abertura à experiência. Assim, por exemplo, no estudo de Jefferson et al. (1998), os 
resultados utilizando dados a respeito da personalidade do indivíduo fornecidos por pessoas próximas apoiaram a hipótese de que não primogênitos apresentam escores mais altos em abertura à experiência (Sulloway, 1995, 1999). Todavia, para dados provenientes de autorrelato, o efeito foi muito pequeno $\left(\eta^{2}=0,01\right)$ (Jefferson et al., 1998). Em virtude disso, delineamentos com medidas colaterais de personalidade podem tornar mais explícito o efeito da ordem do nascimento e suas possíveis diferenças entre homens e mulheres.

$\mathrm{O}$ presente estudo avaliou relações entre a ordem do nascimento e o traço de personalidade abertura à experiência. A investigação, de caráter exploratório, buscou estabelecer uma análise do possível papel moderador do sexo na relação entre a ordem do nascimento e a personalidade. Embora a amostra investigada tenha sido suficiente para a discussão proposta, ressalta-se a importância de estudos mais amplos para investigar uma questão que ainda gera controvérsias no que se refere ao próprio estudo da personalidade humana.

\section{REFERÊNCIAS}

Abramovitch, R., Corter, C., Pepler, D., \& Stanhope, L. (1986). Sibling and peer interaction: A final follow-up and a comparison. Child Development, 57, 217-229

Adler, A. (1964). Problems of neurosis. New York: Harper and Row.

Beer, J. M., \& Horn, J. M. (2000). The influence of rearing order on personality development within two adoption cohorts. Journal of Personality, 68, 789.

Bouchard, T. J., \& Loehling, J. C. (2001). Genes, evolutions, and personality. Behavior Genetics, 31(3), 243273.

Dunkel, C. S., Harbke, C. R., \& Papini, D. R. (2009). Direct and Indirect Effects of Birth Order on Personality and Identity: Support for the Null Hypothesis. The Journal of Genetic Pyschology, 170, 159-175.

Harris, J. R. (2000). Context-specific learning, personality, and birth order. Current Directions in Psychological Science, 9, 174-177.

Harris, J. R. (2007). Não há dois iguais: Natureza humana e individualidade. São Paulo: Globo.
Hutz, C., Nunes, C. H. S. S., Silveira, A. D., Serra, J., Anton, M., \& Wieczorek, L. S. (1998). O desenvolvimento de marcadores para a avaliação da personalidade no modelo dos Cinco Grandes Fatores. Psicologia: Reflexão e Crítica, 11, 171-182.

Magalhães, M. O. (2009). Relação entre ordem de nascimento e estilos interpessoais. Interação em Psicologia, $13,1-11$.

Jefferson, T. J., Herbst, J. H., \& McCrae, R. R. (1998). Associations of birth-order and personality traits: evidence from self-reports and observer ratings. Journal of Research in Personality, 32, 498-509.

Sampaio, I. A. (2007). Práticas educativas parentais, gênero e ordem de nascimento dos filhos: Atualização. Revista Brasileira de Crescimento e Desenvolvimento Humano, 17, 144-152.

Sampaio, I. A., \& Vieira, M. L. (2009) Autodescrição de adolescentes segundo gênero e ordem de nascimento. Psicologia Ciência e Profissão, 29, 574-587.

Skinner, N. F. (2003). Birth-order effects in dominance: faliure to support Sulloway's view. Psychological Reports, 92, 387-388.

Sulloway, F. J. (1999). Vocação Rebelde: Ordem de nascimento, dinâmica familiar e vida criativa. Rio de Janeiro: Record.

Sulloway, F. J. (1995). Birth Order and Evolutionary Psychology: A Meta-Analytic Overview. Psychological Inquiry, 6, 75-80.

Tavares, M. B., Fuchs, F. C., Diligenti, F. T., de Abreu, J. R. P., Rodhe, L. A., \& Fuchs, S. C. (2004). Behavioral characteristics of the only child vs first-born and children with siblings. Revista Brasileira de Psiquiatria, 26, 1723.

Vasconcellos, S. J. L., \& Hutz, C. S. (2008). Construção e validação de uma escala de abertura à experiência. Avaliação Psicológica, 7, 135-141. 